\title{
Quantum Fields and Probability
}

\author{
Antti Kupiainen*
}

\begin{abstract}
I review some recent work where ideas and methods from Quantum Field Theory have proved useful in probability and vice versa. The topics discussed include the use of Renormalization Group theory in Stochastic Partial Differential Equations driven by space-time white noise and the use of the theory of Gaussian Multiplicative Chaos in the study of two dimensional Liouville Conformal Field theory.
\end{abstract}

2010 Mathematics Subject Classification. Primary 81T08; ; Secondary 60H15.

Keywords. Quantum Fields, Stochastic PDE's, Multiplicative Chaos, Liouville Gravity

\section{Quantum Fields and Random Fields}

Quantum Field Theory (QFT) was originally developed as a quantum theory of physical systems with infinite number of degrees of freedom. Perhaps the simplest example is obtained from the wave equation

$$
\left(\partial_{t}^{2}-\Delta\right) \varphi=0
$$

where $\varphi(t, x), x \in \mathbb{R}^{d-1}$. In its first order form $\dot{\varphi}=\pi, \dot{\pi}=\Delta \varphi$ it is an infinite dimensional Hamiltonian system on the phase space of suitable functions $\varphi(x), \pi(x)$. In quantum theory the field $\varphi(t, x)$ becomes an operator valued distribution i.e. the smeared fields $\varphi(f)$ with $f \in \mathcal{S}\left(\mathbb{R} \times \mathbb{R}^{d-1}\right.$ ) act as (unbounded) operators in a Hilbert space $\mathcal{H}$. The physical content of this QFT is summarized by the the Wightman functions

$$
\left(\Omega, \varphi\left(f_{1}\right) \ldots \varphi\left(f_{n}\right) \Omega\right)=\int W_{n}\left(z_{1}, \ldots, z_{n}\right) \prod f\left(z_{i}\right) d z_{i}
$$

where we denoted $(t, x)$ by $z$ and $\Omega \in \mathcal{H}$ is a special vector ("vacuum"). The Wightman functions are distributions, $W_{n} \in S^{\prime}\left(\mathbb{R}^{n d}\right)$ and more generally, an axiomatic characterization of QFT can be given in terms of such distributions [22].

In this formulation there is nothing random about QFT. However, it was later realized that the Wightman functions $W_{n}$ are boundary values of analytic functions in complex $z_{i}$ and they have in particular an analytic continuation to the Euclidean region of imaginary time, leading to Schwinger functions

$$
S_{n}\left(\left(t_{1}, x_{1}\right), \ldots,\left(t_{n}, x_{n}\right)\right):=W_{n}\left(\left(-i t_{1}, x_{1}\right), \ldots,\left(-i t_{n}, x_{n}\right)\right) .
$$

\footnotetext{
${ }^{*}$ Supported by Academy of Finland
} 
The Schwinger functions have a probabilistic interpretation as correlation functions of a random field $\phi(x), x \in \mathbb{R}^{d}$ (modulo a regularity assumption guaranteeing solution of a moment problem):

$$
S_{n}\left(f_{1}, \ldots, f_{n}\right)=\mathbb{E}\left(\phi\left(f_{1}\right) \ldots \phi\left(f_{n}\right)\right) .
$$

In our simple example of quantization of the wave equation the resulting random field is the Gaussian Free Field (GFF), a Gaussian random distribution with covariance operator given by the Green function of the Laplace operator in $\mathbb{R}^{d}$.

Conversely Osterwalder and Schrader [17. showed that one can also do the opposite: starting from the Schwinger functions one can reconstruct the Wightman functions provided the former satisfy a positivity condition called Reflection Positivity. In this sense the subject of QFT can be viewed as a special case of that of random fields.

In fact, this reconstruction of the QFT plays very little role in the major physical application of reflection positive random fields namely the theory of critical phenomena. Here the physics takes place in the Euclidean region and the coordinate corresponding to the (imaginary) time above is a spatial variable. Examples are condensed matter systems which exhibit scale invariance at second order phase transition points. This scale invariance is universal i.e. independent on most microscopic details and it described by Euclidean QFT's.

Of course in QFT one is not interested in the free field but one with interactions. In our wave equation example interactions enter as nonlinear terms in the equation. The resulting random fields are non-gaussian and the goal of Constructive Field Theory is to give examples of such fields satisfying the OS axioms. In the context of critical phenomena the statistics of the fields at the phase transition point are strongly nongaussian in the interesting cases and the scaling properties of the correlation functions differ radically from that of a Gaussian theory.

The construction of such non-gaussian random fields is by no means easy. For example if we add a simple nonlinear term $\varphi^{3}$ to the wave equation then for all $d$ one needs to perform a renormalization: the equation has to be regularized by smoothening it on scale $\epsilon$ and renormalized by adding terms blowing up as $\epsilon \rightarrow 0$. The way to understand this and to find the renormalizations was conceived first by physicists in the 40's studying the QFT in a formal perturbation expansion in the nonlinearity and conceptualized by K. Wilson in the 60's [24] in the theory of Renormalization Group.

Another vast field where ideas from QFT have proved to be very useful deals with noisy dynamics. Stationary states of Markov processes with infinite dimensional state space give rise to QFT-like random fields. Examples are nonlinear Stochastic PDE's driven by space-time white noise and scaling limits of interacting particle systems. These stationary states are usually not OS-positive but nevertheless share many features of QFT's, in particular the problem of renormalization.

In this presentation I will discuss two examples of interaction between QFT and Probability. The first deals with Nonlinear Parabolic PDE's driven by a very rough noise. These equations require renormalization in order to be well posed and QFT ideas are very useful in understanding of this. 
The second example is from Constructive QFT: I will discuss how ideas from the probabilistic theory of multiplicative chaos can be used to construct an interesting Conformal Field Theory, the Liouville Theory which is conjectured to be a basic building block of two dimensional quantum gravity or in other words the scaling limit of discrete two dimensional random surfaces.

\section{Rough SPDE's}

Nonlinear parabolic PDE's driven by a space time decorrelated noise are ubiquitous in physics. These equations are of the form

$$
\partial_{t} \phi=\Delta \phi+V(\phi)+\xi
$$

where $\phi(t, x)$ is defined on $\Lambda \subset \mathbb{R}^{d}, V(\phi)$ is a function of $\phi$ and possibly its derivatives which can also be non-local and $\xi$ is white noise on $\mathbb{R} \times \Lambda$, formally

$$
\mathbb{E} \xi\left(t^{\prime}, x^{\prime}\right) \xi(t, x)=\delta\left(t^{\prime}-t\right) \delta\left(x^{\prime}-x\right)
$$

Examples are the KPZ equation with $d=1$ and

$$
V(\phi)=\left(\partial_{x} \phi\right)^{2} \quad(\mathrm{KPZ})
$$

describing random deposition in surface growth and the Ginzburg-Landau model

$$
V(\phi)=-\phi^{3}
$$

describing stochastic dynamics of spin systems.

Usually in these problems one is interested in the behavior of solutions in large time and/or long distances in space. In particular one is interested in stationary states and their scaling properties. These can be studied with regularized versions of the equations where the noise is replaced by a mollified version that is smooth in small scales. Often one expects that the large scale behavior is insensitive to such regularization.

From the mathematical point of view and sometimes also from the physical one it is of interest to inquire about the short distance properties i.e. about the well-posedness of the equations without regularizations. Then one is encountering the problem that the solutions are expected to have very weak regularity, they are distributions, and it is not clear how to set up the solution theory for the nonlinear equations in distribution spaces.

Recently this problem was addressed by Martin Hairer 10, 11 who set up a solution theory for a class of such equations, including the KPZ equation and the GL equation in three dimensions. The latter case was also addressed by Catellier and Chouk [2] based on the theory of paracontrolled distributions developed in [8]. I will discuss an alternative approach [14, 15, to this problem based on Wilson's RG ideas developed originally in QFT. 
2.1. Divergences. To see what the problem with equations (1) is consider first the linear case $F=0$. We take the spatial domain to be the unit torus $\Lambda=\mathbb{T}^{d}=$ $(\mathbb{R} / \mathbb{Z})^{d}$. The solution with initial data $\phi_{0}$ is then given by

$$
\phi(t, x)=\left(e^{t \Delta} \phi_{0}\right)(x)+\eta(t, x)
$$

with

$$
\eta(t)=\int_{0}^{t} e^{(t-s) \Delta} \xi(s) d s
$$

and we denote the heat semigroup by $e^{t \Delta} \cdot \eta(t, x)$ is a random field with covariance

$$
\mathbb{E} \eta(t, x) \eta(t, y)=C_{t}(x, y)
$$

where $C_{t}(x, y)$ is the integral kernel of the operator

$$
\int_{0}^{t} e^{2 t \Delta} d t=-\frac{1}{2}\left(1-e^{2 t \Delta}\right) \Delta^{-1}
$$

so $\eta$ becomes the GFF as $t \rightarrow \infty$. In particular $C_{t}(x, y)$ is singular in short scales :

$$
\mathbb{E} \eta(t, x) \eta(t, y) \asymp \frac{1}{|x-y|^{d-2}} .
$$

Let now $V \neq 0$ and write (1) as an integral equation (take $\phi_{0}=0$ )

$$
\phi(t)=\int_{0}^{t} e^{(t-s) \Delta}(V(\phi(s))+\xi(s)) d s=\eta(t)+\int_{0}^{t} e^{(t-s) \Delta} V(\phi(s)) d s
$$

where $\eta(t, x)$ is the solution to the linear equation. Fix a realization of the random field $\eta(t, x)$ and try to solve this fixed point problem by Picard iteration

$$
\phi(t)=\eta(t)+\int_{0}^{t} e^{(t-s) \Delta} V(\eta(s)) d s+\ldots
$$

This fails: for the KPZ equation $V(\eta(s))=\left(\partial_{x} \eta(s, x)\right)^{2}$ is not defined as $\eta$ has the regularity of Brownian motion in $x$. For the GL equation $V(\eta(s))=\eta(s, x)^{3}$ and by (3) this is not defined as a random filed as $\mathbb{E} \eta(s, x)^{3} \eta\left(s^{\prime}, x^{\prime}\right)^{3}=\infty$.

2.2. Superrenormalizable QFT. Such divergencies are familiar from QFT. Indeed, in the $V=0$ case the equation has a stationary measure $\mu_{G F F}$ (obtained as $t \rightarrow \infty$ from (3) ) which is the Gaussian Free Field corresponding to the quantization of the linear wave equation discussed in Section 1 . Formally the GL equation then has a stationary measure

$$
\nu(d \phi)=e^{-\frac{1}{4} \int_{\mathbb{T}^{d}} \phi(x)^{4} d x} \mu_{G F F}(d \phi) .
$$

This is precisely the Euclidean QFT measure corresponding to the quantization of the nonlinear wave equation. Since $\phi(x)^{4}$ is not a well defined random variable in $d>1$ to define this measure one needs renormalization. First we regularize

$$
\phi_{\epsilon}(x):=\left(\rho_{\epsilon} * \phi\right)(x), \quad \rho_{\epsilon}(x)=\epsilon^{-d} \rho(x / \epsilon)
$$


where $\rho$ is a smooth mollifier and then renormalize by adding a counter term

$$
U^{(\epsilon)}\left(\phi_{\epsilon}\right):=\frac{1}{4} \phi_{\epsilon}^{4}+\frac{1}{2} r_{\epsilon} \phi_{\epsilon}^{2}
$$

Then

$$
\lim _{\epsilon \rightarrow 0} e^{-\int_{\Lambda} U^{(\epsilon)}\left(\phi_{\epsilon}(x)\right) d x} \mu_{G F F}(d \phi)
$$

exists provided we take

$$
r_{\epsilon}= \begin{cases}m \log \epsilon & d=2 \\ m_{1} \epsilon^{-1}+m_{2} \log \epsilon & d=3\end{cases}
$$

with suitable $m, m_{1}, m_{2}$. Construction of this limit was a major accomplishment of Constructive Field Theory in the 1970's (for references see [20, 9]).

2.3. Counterterms for PDE. Let us take the same approach to the equation (1) by considering a regularized version:

$$
\partial_{t} \phi=\Delta \phi+V_{\epsilon}(\phi)+\xi_{\epsilon}
$$

with mollified nois $\ell^{1} \xi_{\epsilon}(t)=\rho_{\epsilon} * \xi(t)$ and renormalized $V_{\epsilon}$ which has $\epsilon$-dependent terms added to $V$. The noise $\xi_{\epsilon}$ is a.s. smooth so (5) is well posed with a.s. smooth solution $\phi_{\epsilon}$. Our task is to determine $V_{\epsilon}$ so that $\phi_{\epsilon}$ converges as $\epsilon \rightarrow 0$ to some distribution $\phi$. We consider the GL and the KPZ equations and the following generalization of the KPZ equation which shows up in fluctuating hydrodynamics [21] and turns out to be instructive:

$$
\partial_{t} \phi_{\alpha}=\partial_{x}^{2} \phi_{\alpha}+\sum_{\beta \gamma} M_{\alpha}^{\beta \gamma} \partial_{x} \phi_{\beta} \partial_{x} \phi_{\gamma}+c_{\alpha, \epsilon}+\xi_{\epsilon}
$$

where the field $\phi=\left(\phi_{1}, \phi_{2}, \phi_{3}\right)$ takes values in $\mathbb{R}^{3}$ and the coefficients $M_{\alpha}^{\beta \gamma} \in \mathbb{R}$ may be quite general. For this equation we take a constant counter term

$$
c_{\alpha, \epsilon}=a_{\alpha} \epsilon^{-1}+b_{\alpha} \log \epsilon
$$

and for the GL equation we take the counter term $r_{\epsilon} \phi$ with $r_{\epsilon}$ given by (4). Then

Theorem 2.1. There exist constants $a_{\alpha}, b_{\alpha}, m, m_{1}, m_{2}$ s.t. the following holds almost surely in $\xi$ : There exists $T>0$ s.t. the regularized equation has a unique solution $\phi_{\epsilon}(t, x)$ for $t \leq T$ and

$$
\phi_{\epsilon} \rightarrow \phi \in \mathcal{D}^{\prime}\left([0, T] \times \mathbb{T}^{d}\right)
$$

where $\phi$ is independent of the cutoff function $\rho$.

We remark that in general $b_{\alpha} \neq 0$ but for the KPZ case it vanishes.

\footnotetext{
${ }^{1}$ In the RG setup a space time mollification is actually more natural, see [14]
} 
2.4. Perturbative vs. Wilsonian approach. The fixed point problem related to (5) is

$$
\phi(t)=\int_{0}^{t} e^{(t-s) \Delta}\left(V_{\epsilon}(\phi(s))+\xi_{\epsilon}(s)\right) d s .
$$

For $\epsilon>0$ this problem has smooth solution $\phi_{\epsilon}$ at least for some time since the noise is a.s. smooth. However, since the limit $\phi$ will be a distribution its not clear how to set this up as a Banach fixed point problem

The approach in [8, 2, 11] is to develop a nonlinear theory of distributions allowing to formulate and solve the fixed point problem. This can be compared to perturbative renormalization theory in QFT.

Our approach uses another approach to renormalization pioneered by K. Wilson in the 60's 24. In Wilson's approach adapted to the SPDE one would not try to solve equation (1), call it $\mathcal{E}$, directly but rather go scale by scale starting from the scale $\epsilon$ and deriving effective equations $\mathcal{E}_{n}$ for larger scales $L^{n} \epsilon:=\epsilon_{n}, n=1,2, \ldots$ where $L>1$ is arbitrary. Going from scale $\epsilon_{n}$ to $\epsilon_{n+1}$ is a problem with $\mathcal{O}(1)$ cutoff when transformed to dimensionless variables. This problem can be studied by a standard Banach fixed point method.

The possible singularities of the original problem are present in the large $n$ behavior of the corresponding effective equation. One views $n \rightarrow \mathcal{E}_{n}$ as a dynamical system and attempts to find an initial condition at $n=0$ i.e. modify $\mathcal{E}$ so that if we fix the scale $\epsilon_{n}=\epsilon^{\prime}$ and then let $\epsilon \rightarrow 0$ (and as a consequence $n \rightarrow \infty$ ) the effective equation at scale $\epsilon^{\prime}$ has a limit. It turns out that controlling this limit for the effective equations allows one then to control the solution to the original equation (1).

In this approach no new theory of distributions needed and it provides a general method to derive counterterms for subcritical nonlinearities as well as a general method to study universality.

2.5. Dimensionless variables. For simplicity of exposition we will use a regularization in time instead of space in the fixed point problem (6):

$$
\phi(t)=\int_{0}^{t} \chi\left(\frac{t-s}{\epsilon^{2}}\right) e^{(t-s) \Delta}\left(V_{\epsilon}(\phi(s))+\xi(s)\right) d s .
$$

where $\chi$ is smooth, vanishing in a neighborhood of 0 and $\chi(s)=1$ for $s>1$. This cutoff has the same effect as the mollification i.e. regularizing the problem in spatial scales $<\epsilon$.

It will be useful to introduce dimensionless variables in terms of which the cutoff $\epsilon=1$. Define a space time scaling operation $s_{\mu}$ by

$$
\left(s_{\mu} \phi\right)(t, x):=\mu^{\frac{d-2}{2}} \phi\left(\mu^{2} t, \mu x\right) .
$$

This scaling preserves the linear equation $\dot{\phi}=\Delta \phi+\xi$. We will now set

$$
\varphi:=s_{\epsilon} \phi .
$$


Then the KPZ and GL nonlinear terms

$$
V_{\epsilon}(\phi)=\left\{\begin{aligned}
\left(\partial_{x} \varphi\right)^{2}+c_{\epsilon} & \mathrm{KPZ} \\
\varphi^{3}+r_{\epsilon} \varphi & \mathrm{GL}
\end{aligned}\right.
$$

become

$$
v^{(\epsilon)}(\varphi)=\left\{\begin{aligned}
\epsilon^{\frac{2-d}{2}}\left(\partial_{x} \varphi\right)^{2}+\epsilon^{2} c_{\epsilon} & \mathrm{KPZ} \\
\epsilon^{4-d} \varphi^{3}+\epsilon^{2} r_{\epsilon} \varphi & \mathrm{GL}
\end{aligned}\right.
$$

and the fixed point problem (7) becomes

$$
\varphi(t)=\int_{0}^{t} \chi(t-s) e^{(t-s) \Delta}\left(v^{(\epsilon)}(\varphi(s))+\xi(s)\right) d s:=G\left(v^{(\epsilon)}(\varphi)+\xi\right) .
$$

In this dimensionless formulation the equation has cutoff on unit scale (instead of scale $\epsilon$ ) and the nonlinearity is small if $d<2$ (KPZ), $d<4$ (GL). These are the subcritical cases. However, $\varphi$ is now defined on $\left[0, \epsilon^{-2} T\right] \times\left(\epsilon^{-1} \mathbb{T}\right)^{d}$ i.e. we need to control arbitrary large times and volumes as $\epsilon \rightarrow 0$ (we denote the noise in (8) again by $\xi$ : it equals in law the space time white noise on $\left.\left[0, \epsilon^{-2} T\right] \times\left(\epsilon^{-1} \mathbb{T}\right)^{d}\right)$.

2.6. Renormalization Group. Fix now a scale $L>1$ and solve the equation (8) for spatial scales $\in[1, L]$ ( temporal scales $\in\left[1, L^{2}\right]$ ). Concretely, we insert

$$
\chi(t-s)=\chi\left(L^{-2}(t-s)\right)+\left(1-\chi\left(L^{-2}(t-s)\right)\right.
$$

in (8) so that

$$
G=G_{0}+G_{1}
$$

where $G_{0}$ involves scales $\in[1, L]$ and $G_{1}$ scales $\in\left[L, \epsilon^{-1}\right]$. The problem (8) is equivalent to

$$
\varphi=\varphi_{0}+\varphi_{1}
$$

with

$$
\begin{aligned}
& \varphi_{0}=G_{0}\left(v^{(\epsilon)}\left(\varphi_{0}+\varphi_{1}\right)+\xi\right) \\
& \varphi_{1}=G_{1}\left(v^{(\epsilon)}\left(\varphi_{0}+\varphi_{1}\right)+\xi\right)
\end{aligned}
$$

It turns out that (10) is easy to solve: it has time $\mathcal{O}\left(L^{2}\right)$, noise is smooth and nonlinearity is small. The solution $\varphi_{0}$ is a function of $\varphi_{1}: \varphi_{0}=\varphi_{0}(\varphi)$. Inserting this to large scale equation (11) get

$$
\varphi_{1}=G_{1}\left(v^{(\epsilon)}\left(\varphi_{1}+\varphi_{0}\left(\varphi_{1}\right)\right)+\xi\right)
$$

This equation has scales $\geq L$. The final step consists of rescaling back to scales $\geq 1$. Define the scaling transformation by

$$
(s \varphi)(t, x):=L^{\frac{2-d}{2}} \varphi\left(L^{-2} t, L^{-1} x\right) .
$$


and set

$$
\varphi^{\prime}:=s^{-1} \varphi_{1} .
$$

By simple change of variables we have $s^{-1} G s=L^{2} G$ and $s \xi \stackrel{\text { law }}{=} L^{2} \xi$ which lead to a renormalized equation for $\varphi^{\prime}$ :

$$
\varphi^{\prime}=G\left(v^{\prime}\left(\varphi^{\prime}\right)+\xi\right)
$$

where

$$
v^{\prime}\left(\varphi^{\prime}\right)=L^{2} s^{-1} v^{(\epsilon)}\left(s \varphi^{\prime}+\varphi_{0}\left(s \varphi^{\prime}\right)\right)
$$

This is of the same form as the original equation except that $\varphi^{\prime}(t, x)$ has $t \in$ $\left[0, \epsilon^{\prime-2} T\right]$ and $x \in\left(\epsilon^{\prime-1} \mathbb{T}\right)^{d}$ with $\epsilon^{\prime}=L \epsilon$ and the nonlinearity has changed to $v^{\prime}$. The map

$$
\mathcal{R}: v^{(\epsilon)} \rightarrow v^{\prime}:=\mathcal{R} v^{(\epsilon)}
$$

is the Renormalization Group map. Iterating this procedure we obtain a sequence of nonlinearities $\mathcal{R}^{n} v^{(\epsilon)}$ and equations

$$
\varphi=G\left(\mathcal{R}^{n} v^{(\epsilon)}(\varphi)+\xi\right)
$$

whose solution $\varphi$ describes solution of original PDE on scales $\geq L^{n} \epsilon$. Indeed, the iteration of the equation (9) leads to the construction of the solution to the original equation (7) in terms of the one of (14).

We can now address the $\epsilon \rightarrow 0$ limit. Let us define the effective equation for scales $\geq \mu$ by

$$
v_{\mu}^{(\epsilon)}:=\mathcal{R}^{\log (\mu / \epsilon)} v^{(\epsilon)}
$$

We try to fix the counter terms so that for all $\mu$ the following limit exists:

$$
v_{\mu}:=\lim _{\epsilon \rightarrow 0} v_{\mu}^{(\epsilon)}
$$

2.7. Linerization. The RG map $\mathcal{R}(13)$ is a composition of a two maps

$$
\mathcal{R}=\mathcal{S} \circ \mathcal{T}
$$

where $\mathcal{S}$ is the scaling

$$
v(\varphi) \rightarrow(\mathcal{S} v)(\varphi)=L^{2} s^{-1} v(s \varphi)
$$

and $\mathcal{T}$ is a translation

$$
v(\varphi) \rightarrow v(\varphi+\psi)
$$

where $\psi$ is a random function of $v$ solved from the short time problem

$$
\psi=G_{0}(v(\varphi+\psi)+\xi) .
$$

Let $\mathcal{L}$ be the linearization of $\mathcal{R}: \mathcal{R} v=\mathcal{L} v+\mathcal{O}\left(v^{2}\right)$. Since to first order in $v$ we have from (16) $\psi=G_{0} \xi+\mathcal{O}(v)$ we get

$$
(\mathcal{L} v)(\varphi)=(\mathcal{S} v)\left(\varphi+G_{0} \xi\right)
$$


The scaling operator $\mathcal{S}$ has local eigenfunctions

$$
\begin{gathered}
\mathcal{S} \varphi^{k}=L^{\alpha_{k}} \varphi^{k}, \quad \alpha_{k}=2-(k-1) \frac{d-2}{2} \\
\mathcal{S}(\nabla \varphi)^{k}=L^{\beta_{k}}(\nabla \varphi)^{k}, \quad \beta_{k}=2-\frac{k+1}{2} \quad d=1
\end{gathered}
$$

The $\alpha_{k}>0$ (relevant) eigenfunctions expand under $\mathcal{L}, \alpha_{k}<0$ (irrelevant) ones contract. For GL the relevant ones are $\phi^{k}, k \leq 4-d$ and for $\operatorname{KPZ}(\nabla \phi)^{k}, k \leq 2$. Iterating one obtains for GL in $d=3$

$$
\mathcal{L}^{n} \varphi^{3}=L^{n}\left(\varphi+\eta_{L^{-n}}\right)^{3}
$$

and for KPZ

$$
\mathcal{L}^{n}(\nabla \varphi)^{2}=L^{\frac{n}{2}}\left(\nabla \varphi+\nabla \eta_{L^{-n}}\right)^{2}
$$

The random field $\eta_{L^{-n}}$ is a sum of contributions from $n$ scales and in fact it is given by the GFF (17) with small scale cutoff $L^{-n}$ :

$$
\eta_{L^{-n}}(t)=\int_{0}^{t}\left(\chi\left(L^{2 n}(t-s)\right)-\chi(t-s)\right) e^{(t-s) \Delta} \xi(s) d s
$$

In particular

$$
\mathbb{E}\left(\nabla \eta_{L^{-n}}(t, x)\right)^{2} \sim L^{n}
$$

and

$$
\mathbb{E}\left(\eta_{L^{-n}}(t, x)\right)^{2} \sim\left\{\begin{array}{rr}
\log L^{n} & d=2 \\
L^{n} & d=3
\end{array}\right.
$$

For KPZ in linear approximation effective equation becomes

$$
v_{\mu}^{\epsilon}=\mu^{\frac{1}{2}}\left(\nabla \varphi+\nabla \eta_{\epsilon / \mu}\right)^{2}+\mu^{2} c_{\epsilon}
$$

and for GL one gets

$$
v_{\mu}^{\epsilon}=\mu^{4-d}\left(\varphi+\eta_{\epsilon / \mu}\right)^{3}+\mu^{2} r_{\epsilon} \varphi
$$

Due to (18) and (19) these have no limit as $\epsilon \rightarrow 0$.

Why did this happen? For KPZ the nonlinearity $(\nabla \varphi)^{2}$ is relevant with exponent $\frac{1}{2}$ but has size $\epsilon^{\frac{1}{2}}$ which reproduces under iteration. However $\mathcal{R}$ produces a more relevant term, constant in $\varphi$ with exponent $\frac{3}{2}$ and size $\epsilon^{\frac{1}{2}}$. This expands under iteration to $\left(\frac{\mu}{\epsilon}\right)^{\frac{3}{2}} \epsilon^{\frac{1}{2}}=\mathcal{O}\left(\epsilon^{-1}\right)$. The solution is obvious: fix the constant $c_{\epsilon}$ so as to cancel the divergence

$$
c_{\epsilon}=\mathbb{E}\left(\nabla \eta_{\epsilon}\right)^{2}=a \epsilon^{-1}
$$

Then the effective equation becomes

$$
v_{\mu}^{\epsilon}=\mu^{\frac{1}{2}}\left[(\nabla \varphi)^{2}+2 \nabla \varphi \nabla \eta_{\epsilon / \mu}+:\left(\nabla \eta_{\epsilon / \mu}\right)^{2}:\right]
$$


where

$$
:\left(\nabla \eta_{\epsilon / \mu}\right)^{2}:=\left(\nabla \eta_{\epsilon / \mu}\right)^{2}-\mathbb{E}\left(\nabla \eta_{\epsilon / \mu}\right)^{2}
$$

For the GL equation $\mathcal{R}$ produces a relevant linear term in $\varphi$ with exponent 2 . Taking

$$
r_{\epsilon}=\mathbb{E} \eta_{\epsilon}^{2}
$$

the effective equation becomes

$$
v_{\mu}^{\epsilon}=\mu^{4-d}\left[\varphi^{3}+3 \varphi^{2} \eta_{\epsilon / \mu}+3 \varphi: \eta_{\epsilon / \mu}^{2}:+: \eta_{\epsilon / \mu}^{3}:\right]
$$

The limits

$$
\begin{gathered}
\lim _{\epsilon \rightarrow 0}:\left(\nabla \eta_{\epsilon / \mu}(t, x)\right)^{2}:=:(\nabla \eta(t, x))^{2}: \\
\lim _{\epsilon \rightarrow 0}: \eta_{\epsilon / \mu}(t, x)^{k}:=: \eta(t, x)^{k}:
\end{gathered}
$$

are distribution valued random fields, the Wick powers of the GFF. Hence in the linear approximation to the RG the limit (15) exists a.s. as a distribution.

2.8. Outline of the proof. Let us start with GL in $d=2$. Denote the result of the linear approximation by

$$
u_{\mu}^{\epsilon}=\mu^{2}:\left(\varphi+\eta_{\epsilon / \mu}\right)^{3}:
$$

and write

$$
v_{\mu}^{\epsilon}=u_{\mu}^{\epsilon}+w_{\mu}^{\epsilon}
$$

Since $\mathcal{L} u_{\mu}^{\epsilon}=u_{L \mu}^{\epsilon}$ we get

$$
w_{L \mu}^{\epsilon}=\mathcal{L} w_{\mu}^{\epsilon}+\mathcal{O}\left(\mu^{4}\right) .
$$

In $d=2$ we expect from the scaling eigenfunction analysis that $\|\mathcal{L}\| \leq C L^{2}$ in a suitable space. Thus we expect

$$
\left\|w_{L \mu}^{\epsilon}\right\| \leq C L^{2}\left\|w_{\mu}^{\epsilon}\right\|+C \mu^{4} .
$$

Suppose, inductively in the scale $\mu$ that we have shown

$$
\left\|w_{\mu}^{\epsilon}\right\| \leq \mu^{2+\delta}, \quad \delta>0
$$

Then

$$
\left\|w_{L \mu}^{\epsilon}\right\| \leq C L^{2} \mu^{2+\delta}+C \mu^{4} \leq(L \mu)^{2+\delta}
$$

provided we take $L>\mathcal{O}(1)$ and $\mu<C(L)$. Thus we can inductively prove (21) for scales $\mu \leq \mu_{0}$. This becomes a proof once we work in a suitable Banach space of $v$ 's. Thus normal ordering suffices to make the PDE well posed.

Now consider $d=3$ :

$$
u_{\mu}^{\epsilon}=\mu:\left(\varphi+\eta_{\epsilon / \mu}\right)^{3}:
$$

and this time $\|\mathcal{L}\| \leq C L^{5 / 2}$ so that

$$
\left\|w_{L \mu}^{\epsilon}\right\| \leq C L^{5 / 2}\left\|w_{\mu}^{\epsilon}\right\|+C \mu^{2}
$$


Since $5 / 2>2$ this is not good! The linear part expands too rapidly compared with the smallness of the nonlinear contributions for the inducive argument to work. The remedy is to compute $v_{\mu}^{\epsilon}$ explicitly to the second order:

$$
v_{\mu}^{\epsilon}=u_{\mu}^{\epsilon}+U_{\mu}^{\epsilon}+w_{\mu}^{\epsilon}
$$

If we could show that the second order term satisfies the bound

$$
\left\|U_{\mu}^{\epsilon}\right\| \leq C \mu^{2}
$$

we would get

$$
\left\|w_{L \mu}^{\epsilon}\right\| \leq L^{5 / 2}\left\|w_{\mu}^{\epsilon}\right\|+C \mu^{3}
$$

and since $5 / 2<3$ we may proceed inductively as in $d=2$ to show

$$
\left\|w_{\mu}^{\epsilon}\right\| \leq \mu^{5 / 2+\delta}, \quad \delta>0 .
$$

for $\mu \leq \mu_{0}$,

However, $\left\|U_{\mu}^{\epsilon}\right\|$ diverges as $\log \epsilon ! U_{\mu}^{\epsilon}$ is a (nonlocal) polynomial in $\varphi$ and $\eta_{\epsilon / \mu}$. We expand $U_{\mu}^{\epsilon}$ in Wiener chaos (i.e. Wick polynomials). The result is

$$
U_{\mu}^{\epsilon}=b \mu^{2} \log (\epsilon / \mu) \varphi+\tilde{U}_{\mu}^{\epsilon}
$$

where $\lim _{\epsilon \rightarrow 0} \tilde{U}_{\mu}^{\epsilon}$ exists as a random field. Hence we learn that we need to add an additional mass counter term to the equation

$$
v^{(\epsilon)}=\epsilon \varphi^{3}+\epsilon^{2}\left(a \epsilon^{-1}+b \log \epsilon\right) \varphi .
$$

In the original PDE this means

$$
\phi^{3} \rightarrow \phi^{3}+\left(a \epsilon^{-1}+b \log \epsilon\right) \phi
$$

Why did this happen? The linear term is relevant in 1st order and neutral (marginal) in 2nd order. Marginal terms can pile up logarithmic divergences upon iteration. The counter term prevents this. We get

$$
v_{\mu}^{(\epsilon)}=\mu \varphi^{3}+\mu^{2}\left(a \mu^{-1}+b \log \mu\right) \varphi+\ldots
$$

Note that this is small as long as $\mu$ is. Nothing is diverging!

In KPZ coupling constant is $\epsilon^{\frac{1}{2}}$ and $\|\mathcal{L}\|=L^{3 / 2}$ so we need to expand $v_{\mu}^{(\epsilon)}$ to 3rd order. By "miracle" 2nd and 3rd order terms have vanishing relevant and marginal terms. The random fields occurring in them have $\epsilon \rightarrow 0$ limits and no new renormalizations are needed. This is not true for the multicomponent KPZ: this is the source of the $\log \epsilon$ constant counter term coming from in third order where constants are marginal.

In this heuristic discussion we have assumed perturbative terms $u_{\mu}^{\epsilon}$ have the obvious bounds in powers of $\mu$. This can not be true since they involve the random fields : $\eta^{k}:,:(\nabla \eta)^{2}$ : etc. These noise fields belong to Wiener chaos of bounded order and their covariance is in a suitable negative Sobolev space. Hypercontractivity 
implies good moment estimates for them and a Borel-Cantelli argument implies that a.s. we can find a $\mu_{0}>$ s.t. $\left\|u_{\mu}^{\epsilon}\right\|$ has a good bound for $\mu<\mu_{0}$. On that event the $\mathcal{R}$ is controlled by a simple application of contraction mapping in a suitable Banach space. The time of existence of the original SPDE is $\mu_{0}^{2}$ and it is a.s. $>0$.

Finally, let us briefly discuss the domain and range of $v_{\mu}^{\epsilon}(\varphi)$. Recall $v_{\mu}^{\epsilon}=$ $v_{\mu}^{\epsilon}(t, x ; \varphi)$ is a function on space time and a nonlinear functional of the field $\varphi$. Consider first its dependence of $(x, t)$. In the GL case the random fields in the perturbative part $v_{\mu}^{(\epsilon)}$ (i.e. fields such as $\eta,: \eta^{2}:$ etc) are distributions which are in $H_{l o c}^{-2}$ in their time dependence and in $H_{l o c}^{-4}$ in their space dependence. This leads us to let $v_{\mu}^{\epsilon}$ take values in $H_{l o c}^{-2,-4}$.

As for the $\varphi$-dependence of $v_{\mu}^{\epsilon}(t, x ; \varphi)$ we need to discuss the domain, i.e. in what space should the argument $\varphi$ be taken. Since $\varphi$ represents the large scale part of the solution we can take $\varphi$ smooth. Explicitly we let

$$
\varphi \in C^{2,4}\left(\left[0, \mu^{-2} T\right] \times \mu^{-1} \mathbb{T}^{d}\right)
$$

We then prove that

$$
v_{\mu}^{\epsilon}: C^{2,4} \rightarrow H_{l o c}^{-2,-4}
$$

is an analytic function in a ball of radius $\mu^{-\alpha}, \alpha>0$.

2.9. Subcritical equations. $\mathrm{KPZ}_{d=1}$ and $\mathrm{GL}_{d<4}$ are subcritical: the dimensionless strength of nonlinearity is small in short scales. Another example is the Sine-Gordon equation

$$
\partial_{t} \phi=\Delta \phi+g \sin (\sqrt{\beta} \phi)+\xi
$$

After normal ordering dimensionless coupling is

$$
\epsilon^{2-\frac{\beta}{8 \pi}} g .
$$

This is subcritical for $\beta<16 \pi$. Here one needs to expand solution to order $k-1$ where $\left(2-\frac{\beta}{8 \pi}\right) k>2$. So $k \rightarrow \infty$ as $\beta \uparrow 16 \pi$. It is a challenge to carry this out for all $\beta<16 \pi$. Hairer and Shen have controlled the case $\beta<\frac{32 \pi}{3}$ [12].

\section{Liouville QFT}

The QFT's discussed in the previous Section are quite simple from the renormalization group point of view: they are superrenormalizable which means that the counter terms can be found without a multi scale analysis by looking at a few orders of perturbation series (Picard iteration above). We will now discuss another QFT, the Liouville model, that can be considered superrenormalizable but which has several interesting features and applications. Its motivation comes from random surface theory and two dimensional quantum gravity. I will discuss work done with F. David, R. Rhodes and V. Vargas to give a rigorous construction of the Liouville model [3, 4, 16]. 
3.1. Random Surfaces. Let $\mathcal{T}_{N}$ be the set of triangulations of the 2 -sphere $S^{2}$ with $N$ faces, three of which are marked. $T \in \mathcal{T}_{N}$ is a graph with topology of $S^{2}$ and each face has three boundary edges. We will consider a two-parameter family of probability measures $\mathbb{P}_{\mu_{0}, \gamma}$ on $\mathcal{T}=\cup_{N} \mathcal{T}_{N}$ defined by

$$
\mathbb{P}_{\mu_{0}, \gamma}(T)=\frac{1}{Z_{\mu_{0}, \gamma}} e^{-\mu_{0} N} Z_{\gamma}(T)
$$

if $T \in \mathcal{T}_{N} . Z_{\gamma}(T)$ is the partition function of a critical lattice model on the graph $T$. Such models are defined for $\gamma \in[\sqrt{2}, 2]$ and some examples are percolation for $\gamma=\sqrt{8 / 3}$, Ising model $\gamma=\sqrt{3}$, discrete GFF for $\gamma=2$, uniform spanning tree $\gamma=\sqrt{2}$. It is known that

$$
Z_{N}:=\sum_{T \in \mathcal{T}_{N}} Z_{\gamma}(T)=N^{1-\frac{4}{\gamma^{2}}} e^{\bar{\mu} N}(1+o(1))
$$

so that $\mathbb{P}_{\mu_{0}, \gamma}$ is defined for $\mu_{0}>\bar{\mu}$. $Z_{\mu_{0}, \gamma}$ diverges as $\mu_{0} \rightarrow \mu$ so that the measure concentrates on large triangulations in that limit.

Each $T$ has a natural conformal structure where each face $f$ is equilateral with unit area. Then there is a unique conformal map $\psi: T \rightarrow S^{2}$ s.t. centers of marked faces map to $z_{1}, z_{2}, z_{3}$. Let $\nu_{T}$ be the image of the area measure on $T$. Under $\mathbb{P}_{\mu_{0}, \gamma}, \nu_{T}$ becomes a random measure $\nu_{\mu_{0}, \gamma}$ on $S^{2}$.

Consider now a scaling limit as follows. Recalling that as $\mu_{0} \downarrow \bar{\mu}$ typical size of triangulation diverges we define for $\mu>0$

$$
\rho_{\mu, \gamma}^{(\epsilon)}:=\epsilon \nu_{\bar{\mu}+\epsilon \mu, \gamma}
$$

so that the image triangles have area $\epsilon$. It is natural to conjecture that $\rho_{\mu, \gamma}^{(\epsilon)}$ converges in law as $\epsilon \rightarrow 0$ to a random measure $\rho_{\mu, \gamma}$. Since $\epsilon \nu_{T}\left(S^{2}\right)=\epsilon N$ the law of $\rho_{\mu, \gamma}^{(\epsilon)}\left(S^{2}\right)$ is given by using (23)

$$
\mathbb{E}\left[F\left(\rho_{\mu, \gamma}^{(\epsilon)}\left(S^{2}\right)\right)\right]=\frac{1}{Z_{\epsilon}} \sum_{N} e^{-\mu \epsilon N} N^{1-\frac{4}{\gamma^{2}}} F(\epsilon N)+o(1) .
$$

Hence this law converges to $\Gamma\left(2-\frac{4}{\gamma^{2}}, \mu\right)$. We will construct a measure with this law for its mass.

3.2. KPZ Conjecture. Let $g(z)|d z|^{2}$ be a smooth conformal metric on the Riemann sphere $\hat{\mathbb{C}}=\mathbb{C} \cup\{\infty\}$. Kniznik, Polyakov and Zamolochicov [13] argued that the random measure $\rho_{\mu, \gamma}$ is given by

$$
\rho_{\mu, \gamma}(d z)=e^{\gamma \phi_{g}(z)} d z
$$

where $\phi_{g}$ is the Liouville field

$$
\phi_{g}:=X+\frac{Q}{2} \ln g
$$


and $X$ is a random field whose law is formally given by

$$
\mathbb{E}_{\gamma, \mu} f(X)=Z^{-1} \int_{M a p(\mathbb{C} \rightarrow \mathbb{R})} f(X) e^{-S_{L}(X, g)} D X .
$$

where $S_{L}$ is action functional of the Liouville model:

$$
S_{L}(X, g):=\frac{1}{\pi} \int_{\mathbb{C}}\left(\partial_{z} X \partial_{\bar{z}} X+\frac{Q}{4} g R_{g} X+\mu e^{\gamma \phi_{g}}\right) d z .
$$

Here $R_{g}=-4 g^{-1} \partial_{z} \partial_{\bar{z}} \log g$ is the scalar curvature and $Q$ is related to $\gamma$ by

$$
Q=2 / \gamma+\gamma / 2 .
$$

Furthermore the heuristic integration over $X$ in (26) is supposed to include "gauge fixing" due to the marked points $z_{1}, z_{2}, z_{3}$.

3.3. GFF. Let us first keep only the quadratic term in the action functional (27) and try to define the linear functional

$$
\langle F\rangle=\int_{M a p(\mathbb{C} \rightarrow \mathbb{R})} F(X) e^{-\frac{1}{4 \pi} \int_{\mathbb{C}}\left|\partial_{z} X\right|^{2} d z} D X
$$

We may define this in terms of the Gaussian Free Field. GFF on the full plane is defined up to constant and we fix this by considering the field $X_{g}$ with zero average in the metric $g$ :

$$
m_{g}\left(X_{g}\right):=\frac{1}{\int_{\mathbb{C}} g(z) d z} \int_{\mathbb{C}} X_{g}(z) g(z) d z=0 .
$$

Then we set $X=X_{g}+c, c \in \mathbb{R}$ and define

$$
\langle F\rangle=\int_{\mathbb{R}}\left(\mathbb{E} F\left(X_{g}+c\right)\right) d c:=\int F(X) d \nu_{G F F}(X) .
$$

Note that $\nu_{G F F}(d X)=\mathbb{P}\left(d X_{g}\right) d c$ is not a probability measure. This measure is independent of the chosen metric since

$$
X_{g^{\prime}} \stackrel{\text { law }}{=} X_{g}-m_{g^{\prime}}\left(X_{g}\right)
$$

where $m_{g^{\prime}}\left(X_{g}\right)$ is a random constant that can be absorbed to a shift in $c$.

We can now give a tentative definition of the measure in (26) by defining

$$
\nu_{g}=e^{-\frac{1}{4 \pi} \int_{\mathbb{C}}\left(Q R_{g} X+\mu e^{\gamma X_{g}}\right) g d z} \nu_{G F F} .
$$

However, now we encounter the problem of renormalization as $e^{\gamma X}$ is not defined since $X_{g}$ is not defined point wise. Indeed $\mathbb{E} X_{g}(z) X_{g}\left(z^{\prime}\right)=\ln \left|z-z^{\prime}\right|^{-1}+\mathcal{O}(1)$ as $z-z^{\prime} \rightarrow 0$. 
3.4. Multiplicative Chaos. To define $e^{\gamma X}$ we proceed as in Section 2 by taking a mollified version of GFF $X_{g, \epsilon}$. Then $\mathbb{E} e^{\gamma X_{g, \epsilon}(z)} \propto \epsilon^{-\frac{\gamma^{2}}{2}}$ and we renormalize by defining thr random measure on $\mathbb{C}$

$$
M_{g, \gamma, \epsilon}(d z):=\epsilon^{\frac{\gamma^{2}}{2}} e^{\gamma\left(X_{g, \epsilon}(z)+\frac{Q}{2} \ln g(z)\right)} d z
$$

Then

$$
M_{g, \gamma, \epsilon} \rightarrow M_{g, \gamma}
$$

weakly in probability as $\epsilon \rightarrow 0$. The limit is nonzero if and only if $\gamma<2$. It is an example of Gaussian multiplicative chaos (see [6] for a review), a random multifractal measure on $\mathbb{C}$ for which a.s. $M_{g, \gamma}(\mathbb{C})<\infty$. We may now define (29) as

$$
\nu_{g}=e^{-\frac{1}{4 \pi}\left(\int_{\mathbb{C}} Q R_{g} X g d z+\mu e^{\gamma c} M_{g, \gamma}(\mathbb{C})\right)} \nu_{G F F}
$$

3.5. Weyl and Möbius invariance. We saw that $X$ is metric independent under $\nu_{G F F}$. Recaling the Liouville field (25) we have

Proposition 3.1. Let $F \in L^{1}\left(\nu_{g}\right)$ and $g^{\prime}=e^{\varphi} g$. Then

$$
\int F\left(\phi_{g^{\prime}}\right) d \nu_{g^{\prime}}=e^{\frac{c_{L}-1}{96 \pi} \int|\partial \varphi|^{2} d z+\int 2 R_{g} \varphi g d z} \int F\left(\phi_{g}\right) d \nu_{g}
$$

where $c_{L}=1+6 Q^{2}$.

Proof. (see 3] for details) By metric independence of $X$ we replace $c+X_{g^{\prime}}$ by $c+X_{g}$ so that

$$
\int F\left(\phi_{g^{\prime}}\right) d \nu_{g^{\prime}}=\int F\left(\phi_{g}+\frac{Q}{2} \varphi\right) e^{\left.-\frac{Q}{4 \pi} \int R_{g^{\prime}} g^{\prime}\left(c+X_{g}\right) d z+\mu e^{\gamma c} \int e^{\frac{Q}{2} \ln \varphi} d M_{g, \gamma}\right)} d \nu_{G F F}
$$

Use $R_{g^{\prime}} g^{\prime}=R_{g} g-\Delta \varphi$ and Gauss-Bonnet theorem $\int R_{g^{\prime}} g^{\prime}=8 \pi=\int R_{g} g$ to get

$$
\int R_{g^{\prime}} g^{\prime}\left(c+X_{g}\right) d z=\int R_{g} g\left(c+X_{g}\right) d z-\int \Delta \varphi X_{g} d z .
$$

Then a shift in the Gaussian integral (Girsanov theorem) completes the proof.

The multiplicative factor is called the Weyl anomaly in physics literature and $c_{L}$ is the central charge of Liouville theory. As a consequence of the Proposition we get Möbius transformation rule (see see [3])

Corollary 3.2. Let $\psi$ be a Möbius map of $\hat{C}$. Then

$$
\int F\left(\phi_{g}\right) d \nu_{g}=\int F\left(\phi_{g} \circ \psi+Q \ln \left|\psi^{\prime}\right|\right) d \nu_{g}
$$


3.6. Vertex operators. Since the Möbius group is non-compact the Corollary makes one suspect that the measure $\nu_{g}$ does not have a finite mass. Indeed, by Proposition 3.1 we may work with the round metric $\hat{g}$ where $R_{\hat{g}}=2$. Then $\frac{1}{4 \pi} \int R_{\hat{g}} \hat{g} d z=2 c$ by Gauss-Bonnet and $\int X_{\hat{g}} \hat{g} d z=0$. We get

$$
\int 1 d \nu_{\hat{g}}=\int \mathbb{E}_{\hat{g}} e^{-2 Q c} e^{-\mu e^{\gamma c} M_{\hat{g}, \gamma}(\mathbb{C})} d c=\infty
$$

as the integral diverges at $c \rightarrow-\infty$ and $M_{\hat{g}, \gamma}(\mathbb{C})<\infty$ a.s..

Recall that we are looking for a measure with three points on $\hat{\mathbb{C}}$ fixed. We define (regularized) vertex operators

$$
V_{\alpha, \epsilon}(z):=\epsilon^{\frac{\alpha^{2}}{2}} e^{\alpha \phi_{\hat{g}, \epsilon}(z)}
$$

and consider their correlation function

$$
\left\langle\prod_{i=1}^{n} V_{\alpha_{i}}\left(z_{i}\right)\right\rangle_{\hat{g}}:=\lim _{\epsilon \rightarrow 0} \int \prod_{i=1}^{n} V_{\alpha_{i}, \epsilon}\left(z_{i}\right) d \nu_{\hat{g}}
$$

Now the $c$-integral converges if and only if $\sum \alpha_{i}>2 Q$ :

$$
\int_{\mathbb{R}} e^{\left(\sum \alpha_{i}-2 Q\right) c-\mu e^{\gamma c} M_{\hat{g}, \gamma}(\mathbb{C})} d c=\gamma^{-1} \mu^{-s} \Gamma(s) M_{\hat{g}, \gamma}(\mathbb{C})^{-s}
$$

with $s=\gamma^{-1}\left(\sum_{i} \alpha_{i}-2 Q\right)$. The remaining expectation over the GFF can be dealt with a shift of $X_{\hat{g}}$ to dispose of $\prod_{i} e^{\alpha_{i} X_{\hat{g}, \epsilon}\left(z_{i}\right)}$. The result is after some calculation $([3,[16])$

$$
\left\langle\prod_{i=1}^{n} V_{\alpha_{i}}\left(z_{i}\right)\right\rangle_{\hat{g}}=\text { const. } \prod_{j<k} \frac{1}{\left|z_{j}-z_{k}\right|^{\alpha_{j} \alpha_{k}}} \mu^{-s} \gamma^{-1} \Gamma(s) \mathbb{E} M_{\hat{g}, \gamma}(F)^{-s}
$$

where

$$
F(z)=\prod_{i} \frac{1}{\left|z-z_{i}\right|^{\gamma \alpha_{i}}} \hat{g}(z)^{-\frac{\gamma}{4} \sum_{l} \alpha_{l}}
$$

The modulus of continuity of the Chaos measure is

$$
M_{\hat{g}}\left(B_{r}\right) \leq C(\omega) r^{\gamma Q-\delta}
$$

for any $\delta>0$. This leads to integrability of $F$ if $\alpha_{i}<Q$ for all $i$ and

Proposition 3.3. $0<\left\langle\prod_{i=1}^{n} V_{\alpha_{i}}\left(z_{i}\right)\right\rangle_{\hat{g}}<\infty$ if and only if $\sum \alpha_{i}>2 Q$ and $\alpha_{i}<Q$.

These bounds for $\alpha_{i}$ are called Seiberg bounds. Note that they imply that we need at at least three vertex operators to have a finite correlation function. 
3.7. KPZ conjectures. Given $z_{1}, z_{2}, z_{3}$ we define the probability measure

$$
d \hat{\mathbb{P}}_{\mu, \gamma}:=\left\langle\prod_{i=1}^{3} V_{\gamma}\left(z_{i}\right)\right\rangle_{\hat{g}}^{-1} \prod_{i=1}^{n} V_{\gamma}\left(z_{i}\right) d \nu_{\hat{g}}
$$

We may now state the KPZ conjecture precisely: the random measure $\rho_{\mu, \gamma}$ coming from scaling limit is in law equal to the measure $\mathcal{M}:=e^{\gamma c} M_{\hat{g}, \gamma}$ under $\hat{\mathbb{P}}_{\mu, \gamma}$. Let $A=\mathcal{M}(\mathbb{C})$ be the "volume of the universe". By a simple change of variables in the $c$-integration $e^{\gamma c} M_{g, \gamma}=A$ we obtain

$$
\mathbb{E} F(A)=\frac{\mu^{s}}{\Gamma(s)} \int_{0}^{\infty} F(y) y^{s} e^{-\mu y} d y
$$

where $s=(3 \gamma-2 Q) / \gamma=2-4 / \gamma^{2}$ i.e. under $\mathbb{P}_{\mu, \gamma}$ the law of $A$ is $\Gamma\left(2-4 / \gamma^{2}, \mu\right)$. which agrees with the result in random surfaces.

The emphasis of KPZ was actually on correlation functions of Conformal Field Theories on random surfaces. As an example, consider the Ising model $(\gamma=\sqrt{3})$. We can transport the Ising spins $\sigma_{v}= \pm 1$ sitting at vertices $v$ of $T$ to $\hat{\mathbb{C}}$. Define the distribution

$$
\Phi_{T}^{(\epsilon)}(z)=\epsilon^{\frac{5}{6}} \sum_{v \in \mathcal{V}(T)} \sigma_{v} \delta\left(z-\psi_{T}(v)\right)
$$

where $\psi_{T}: T \rightarrow \hat{\mathbb{C}}$ is the uniformizing map. Then under $\mathbb{P}_{\mu_{0}+\epsilon \mu, \gamma}$ this becomes a random field on $\hat{\mathbb{C}}$ and the $\mathrm{KPZ}$ conjecture says that its correlation functions converge (in the sense of distributions) to a product form

$$
\lim _{\epsilon \rightarrow 0} \mathbb{E} \Phi^{(\epsilon)}\left(u_{1}\right) \ldots \Phi^{(\epsilon)}\left(u_{n}\right)=\mathbb{E} \sigma\left(u_{1}\right) \ldots \sigma\left(u_{n}\right) \mathbb{E}_{\mu, \gamma} V_{\alpha}\left(u_{1}\right) \ldots V_{\alpha}\left(u_{n}\right)
$$

where $\mathbb{E} \sigma\left(u_{1}\right) \ldots \sigma\left(u_{n}\right)$ are the correlation functions of the Ising model in the scaling limit on $\hat{\mathbb{C}}$ and $\alpha$ is determined from the requirement $\frac{1}{16}+\Delta_{\alpha}=1$ which means that $\sigma(z) e^{\alpha \phi_{g}(z)}$ transforms under conformal maps as a density.

3.8. Conformal Field Theory. So far we have motivated the Liouville model through its conjectural relationship to scaling limits of random triangulations. However, the Liouville model is also an interesting Conformal Field Theory by itself. This way of looking we view the vertex operators as (Euclidean) quantum fields.

First, using the Möbius invariance (Corollary 3.2) of $\nu_{g}$ and taking care with the transformation of the $\epsilon$ in the vertex operator one gets

$$
\left\langle\prod_{i=1}^{n} V_{\alpha_{i}}\left(\psi\left(z_{i}\right)\right)\right\rangle_{g}=\prod_{i}\left|\psi^{\prime}\left(z_{i}\right)\right|^{-2 \Delta_{\alpha_{i}}}\left\langle\prod_{i=1}^{n} V_{\alpha_{i}}\left(z_{i}\right)\right\rangle_{g}
$$

where $\Delta_{\alpha}=\frac{\alpha}{2}\left(Q-\frac{\alpha}{2}\right)$. In CFT parlance, $V_{\alpha}$ is a primary field with conformal weight $\Delta_{\alpha}$. 
Second, the Liouville model has also local conformal symmetry. In CFT this derives from the energy-momentum tensor which encodes the variations of the theory with respect to the background metric. More specifically, one may define the the correlation functions in a smooth Riemannian metric near our $g$ and consider the one parameter family $g_{\epsilon}^{-1}=g^{-1}+\epsilon f \partial_{z} \otimes \partial_{z}$ where $f$ is a smooth function with support in $\mathbb{C} \backslash \cup_{i} z_{i}$. Then (a component of) the stress tensor $T(z)$ is defined by the following formula in the physics literature (see [7])

$$
\left.\frac{d}{d \epsilon}\right|_{\epsilon=0}\left\langle\prod_{l} V_{\alpha_{l}}\left(z_{l}\right)\right\rangle_{g_{\epsilon}}:=\int f(z)\left\langle T(z) \prod_{l} V_{\alpha_{l}}\left(z_{l}\right)\right\rangle_{g} g(z) d z .
$$

A simple formal computation then yields the following heuristic formula

$$
T(z)=Q \partial_{z}^{2} \phi(z)-\left(\left(\partial_{z} \phi(z)\right)^{2}-\mathbb{E}\left(\partial_{z} X_{g}(z)\right)^{2}\right)
$$

where $\phi$ is the Liouville field.In the same way, perturbing the metric instead by $\epsilon f \partial_{\bar{z}} \otimes \partial_{\bar{z}}$ yields the field $\bar{T}(z)$.

$T(z)$ encodes local conformal symmetries through the Conformal Ward Identities. The first Ward identity controls the singularity as the argument of $T$ gets close to one of the $V_{\alpha}$ :

$$
\left\langle T(z) \prod_{l} V_{\alpha_{l}}\left(z_{l}\right)\right\rangle_{g}=\sum_{k} \frac{\Delta_{\alpha_{k}}}{\left(z-z_{k}\right)^{2}}\left\langle\prod_{l} V_{\alpha_{l}}\left(z_{l}\right)\right\rangle_{g}-\sum_{k} \frac{1}{z-z_{k}} \partial_{z_{k}}\left\langle\prod_{l} V_{\alpha_{l}}\left(z_{l}\right)\right\rangle_{g}
$$

and the second identity controls the singularity when two $T$-insertions come close

$$
\begin{aligned}
& \left\langle T(z) T\left(z^{\prime}\right) \prod_{l} V_{\alpha_{l}}\left(z_{l}\right)\right\rangle_{g}=\frac{\frac{1}{2} c_{\mathrm{L}}}{\left(z-z^{\prime}\right)^{4}}\left\langle T\left(z^{\prime}\right) T(z) \prod_{l} V_{\alpha_{l}}\left(z_{l}\right)\right\rangle_{g} \\
& +\frac{2}{\left(z-z^{\prime}\right)^{2}}\left\langle T\left(z^{\prime}\right) \prod_{l} V_{\alpha_{l}}\left(z_{l}\right)\right\rangle_{g}+\frac{1}{z-z^{\prime}} \partial_{z^{\prime}}\left\langle T\left(z^{\prime}\right) \prod_{l} V_{\alpha_{l}}\left(z_{l}\right)\right\rangle_{g}+\ldots
\end{aligned}
$$

where the dots refer to terms that are bounded as $z \rightarrow z^{\prime}$. In [16] we define $T(z)$ rigorously and prove the Ward identities.

3.9. Representation Theory. Let us finally reconstruct the quantum theory from our probabilistic framework. Fix the metric $g=\hat{g}$, the round metric. Let $\mathcal{F}_{\mathbb{D}}$ consist of functions $F(\phi)$ measurable w.r.t. the $\sigma$-algebra generated by $\left.\phi\right|_{\mathbb{D}}$. The measure $\nu_{\hat{g}}$ is reflection positive:

$$
(F, G):=\int \overline{F(X)}(\Theta G)(X) d \nu_{\hat{g}}(X) \geq 0 \quad \forall F, G \in \mathcal{F}_{\mathbb{D}}
$$

where $(\Theta F)(X):=F(\theta X)$ and $(\theta X)(z)=X(1 / \bar{z})$. Define the Physical Hilbert space as (here bar denotes completion)

$$
\mathcal{H}:=\overline{\mathcal{F}_{\mathbb{D}} /\{F:(F, F)=0\}}
$$


The GFF can be decomposed to an independent sum:

$$
X_{\hat{g}}=X_{\mathbb{D}}+X_{\mathbb{D}^{c}}+P \psi
$$

where $X_{\mathbb{D}}$ and $X_{\mathbb{D}^{c}}$ are Dirichlet GFF's on $\mathbb{D}$ and $\mathbb{D}^{c}, \psi$ is the restriction of the GFF to $\partial \mathbb{D}=S^{1}$ with zero average ("1/f noise") and $P \psi$ is the harmonic extension of $\psi$ on $\mathbb{C}$. Let $\mathbb{E}_{\mathbb{D}}$ be the expectation in the $X_{\mathbb{D}}$. Then

$$
(U F)(c, \psi):=e^{-Q c} \mathbb{E}_{\mathbb{D}}\left(e^{-\mu \int_{\mathbb{D}} e^{\gamma \phi} d z} F(\phi)\right)
$$

defines a unitary map

$$
U: \mathcal{H} \rightarrow L^{2}(\mathbb{P}(d \psi) d c)
$$

and we may identify $\mathcal{H}$ with the latter. The dilation $z \rightarrow e^{-t} z$ with $t \geq 0$ acts on $\mathcal{F}_{\mathbb{D}}$ and generates a contraction semigroup

$$
e^{-t H}: \mathcal{H} \rightarrow \mathcal{H}
$$

The generator $H \geq 0$ is the Hamiltonian operator of the CFT.

Let $\mathcal{V}$ be the linear span of the vectors $U\left(\prod_{i=1}^{n} V_{\alpha_{i}}\left(z_{i}\right)\right)$ with $\left|z_{i}\right|<1$. Then

$$
L_{n}=\oint_{|z|=r} z^{n+1} T(z) .
$$

acts on $\mathcal{V}$ by taking $1-r$ small enough. The Ward identities imply the Virasoro algebra commutation rules on $\mathcal{V}$ :

$$
\left[L_{m}, L_{n}\right]=(m-n) L_{m+n}+\frac{c_{L}}{12} m\left(m^{2}-1\right) \delta_{m,-n}
$$

The operators satisfy $L_{n}^{*}=L_{-n}$ on $\mathcal{V}$. The conjugate field $\bar{T}$ gives rise to another copy of the Virasoro algebra. A major challenge is to study the reduction of this representation to irreducibles. It is conjectured 19 that $\mathcal{H}$ decomposes to a direct integral $\int_{\mathbb{R}_{+}}^{\oplus} \mathcal{H}_{P} d P$ where $\mathcal{H}_{P}$ is a highest weight module for the two algebras with $L_{0} \psi_{P}=\Delta_{Q+i P} \psi_{P}$ and similarly for $\bar{L}_{0} . \psi_{P}$ is formally the state corresponding to the vertex operator $V_{Q+i P}$ which saturates the Seiberg bound. In [4] these were constructed for $P=0$. It would be nice to understand the complex case.

3.10. DOZZ-conjecture. In conformal field theory it is believed 1 that all correlation functions are determined by the knowledge of primary fields (i.e. spectrum of representations) and their three point functions. For the latter there is a remarkable conjecture due to Dorn, Otto, Zamolodchikov and Zamolodchikov [5, 25] in Liouville theory. By Möbius invariance

$$
\left\langle V_{\alpha_{1}}\left(z_{1}\right) V_{\alpha_{2}}\left(z_{2}\right) V_{\alpha_{3}}\left(z_{3}\right)\right\rangle=\left|z_{1}-z_{2}\right|^{2 \Delta_{12}}\left|z_{2}-z_{3}\right|^{2 \Delta_{23}}\left|z_{1}-z_{3}\right|^{2 \Delta_{13}} C_{\gamma}\left(\alpha_{1}, \alpha_{2}, \alpha_{3}\right)
$$

where $\Delta_{12}=\Delta_{\alpha_{3}}-\Delta_{\alpha_{1}}-\Delta_{\alpha_{2}}$ etc. and

$$
C_{\gamma}\left(\alpha_{1}, \alpha_{2}, \alpha_{3}\right)=\text { const. } \mu^{-s} \Gamma(s) \mathbb{E} Z^{-s}
$$


with

$$
Z=\int|z|^{-\alpha_{1} \gamma}|z-1|^{-\alpha_{2} \gamma} \hat{g}(z)^{-\frac{\gamma}{4} \sum_{i=1}^{3} \alpha_{i}} M_{\hat{g}, \gamma}(d z) .
$$

The DOZZ Conjecture gives an explicit formula for $C_{\gamma}\left(\alpha_{1}, \alpha_{2}, \alpha_{3}\right)$. It is based on analyticity and symmetry assumptions that lack proofs. One of the ingredients in its derivation was recently proved in [16 namely the so-called $B P Z$ equations [1] for the vertex operators $V_{\chi}$ with $\chi=\left(-\frac{\gamma}{2}\right)^{ \pm 1}$ (in the language of CFT, these are level two degenerate fields). More precisely, we prove

$$
\left(\frac{1}{\chi^{2}} \partial_{z}^{2}+\sum_{k}\left(\frac{\Delta_{\alpha_{k}}}{\left(z-z_{k}\right)^{2}}+\frac{1}{z-z_{k}} \partial_{z_{k}}\right)\right)\left\langle V_{\chi}(z) \prod_{i} V_{\alpha_{i}}\left(z_{i}\right)\right\rangle=0 .
$$

Using the BPZ equation, we recover an explicit formula found earlier in the physics literature for the 4 point correlation function $\left\langle V_{-\frac{\gamma}{2}}(z) \prod_{i=1}^{3} V_{\alpha_{i}}\left(z_{i}\right)\right\rangle$. Following what is called Teschner's trick [23, we then deduce a non trivial functional relation for $C_{\gamma}\left(\alpha_{1}, \alpha_{2}, \alpha_{3}\right)$. The DOZZ formula follows from this relation provided $C_{\gamma}\left(\alpha_{1}, \alpha_{2}, \alpha_{3}\right)$ can be extended analytically away from the region $\sum \alpha_{i}>2 Q$ where it is defined. It is a challenge to complete this argument.

\section{References}

[1] A.A. Belavin, A.M.Polyakov, A.B.Zamolodchikov : Infinite conformal symmetry in two-dimensional quantum field theory, Nuclear Physics B 241 (2)(1984), 333-380 .

[2] R. Catellier and K. Chouk: Paracontrolled distributions and the 3-dimensional stochastic quantization equation. ArXiv: 1310.6869 (2013)

[3] Liouville Quantum Gravity on the Riemann sphere, Communications in Mathematical Physics, 342, (2016), 869-907.

[4] F.David, A.Kupiainen, R.Rhodes, V.Vargas, Renormalizability of Liouville Quantum Gravity at the Seiberg bound, http://arxiv.org/abs/1506.01968.

[5] H.Dorn, H.-J.Otto: Two and three-point functions in Liouville theory, Nucl.Phys. B429 (1994) 375-388

[6] B.Duplantier, R.Rhodes, S.Sheffield, V.Vargas, Log-correlated Gaussian fields: an overview, arXiv:1407.5605

[7] K.Gawedzki, Lectures on conformal field theory. In Quantum fields and strings: A course for mathematicians, 727805. Amer. Math. Soc., Providence, RI, (1999).

[8] M. Gubinelli, P. Imkeller, and N. Perkowski: Paracontrolled distributions and singular PDEs. Forum Math. Pi. 3 (2015)

[9] J. Glimm and A. Jaffe, Quantum Physics. A Functional Integral Point of View (Springer Verlag, Berlin, Heidelberg and New York) 1987.

[10] M. Hairer: Solving the KPZ equation. Ann. Math. 178(2), 559-664 (2013)

[11] M. Hairer: A theory of regularity structures. Invent. Math. 198(2) (2014), 269-504 
[12] M. Hairer, H. Shen, The dynamical sine-Gordon model arXiv:1409.5724 [math.PR]

[13] V.G.Knizhnik, A.M.Polyakov, A.B.Zamolodchikov, Fractal structure of 2D-quantum gravity, Modern Phys. Lett A, 3(8) (1988), 819-826.

[14] A. Kupiainen, Renormalization group and Stochastic PDEs. Ann. Henri Poincar 17(3) (2016), 497-535

[15] A. Kupiainen, M. Marcozzi, Renormalization of Generalized KPZ equation, arXiv:1604.08712

[16] A.Kupiainen, R.Rhodes, V.Vargas, Conformal Ward and BPZ Identities for Liouville quantum field theory, arXiv:1512.01802 [math.PR]

[17] K. Osterwalder and R. Schrader, Axioms for Euclidean Greens functions, II, Commun. Math. Phys., 42 (1975), 281305

[18] A.M.Polyakov, Quantum geometry of bosonic strings, Phys. Lett. 103B 207 (1981).

[19] S.Ribault, Conformal Field theory on the plane, arXiv:1406.4290.

[20] B. Simon, The $P(\phi)_{2}$ - Euclidean (Quantum) Field Theory (Princeton University Press, Princeton) 1974.

[21] H. Spohn: Nonlinear Fluctuating Hydrodynamics for Anharmonic Chains. J. Stat. Phys. 154 (2014), 1191-1227

[22] R.F. Streater and A.S. Wightman, PCT, Spin and Statistics, and All That (Reading, Mass., Benjamin/Cummings Publ. Co.) 1964.

[23] J.Teschner, On the Liouville three point function, Phys. Letters B363 (1995), 65-70.

[24] K. Wilson: The renormalization group and critical phenomena. Nobel Lecture. Rev. Mod. Phys. (1984)

[25] A.B. Zamolodchikov, Al.B. Zamolodchikov, Structure constants and conformal bootstrap in Liouville field theory, Nucl. Phys. B 477, 577-605 (1996).

Antti Kupiainen, Department of Mathematics and Statistics, University of Helsinki, PO Box 68, 00014 Helsinki, Finland

E-mail: Antti.Kupiainen@helsinki.fi 